\title{
Mycobacterial transport medium for routine culture of fine needle aspiration biopsies
}

\author{
C A Wright, A Vermaak \\ Divisions of Anatomical Pathology, Department of Pathology, Stellenbosch University and NHLS Tygerberg \\ Hospital, Tygerberg, South Africa. \\ C Bamford, Y Prince, \\ Medical Microbiology, Department of Pathology, Stellenbosch University and NHLS Tygerberg Hospital, \\ Tygerberg, South Africa. \\ K G P Hoek, R M Warren \\ NRF Centre of Excellence in Biomedical Tuberculosis Research/MRC Centre for Molecular and Cellular \\ Biology, Division of Molecular Biology and Human Genetics, Stellenbosch University, Stellenbosch, South \\ Africa. \\ B J Marais \\ Department of Pediatrics and Child Health, Stellenbosch University, Stellenbosch, South Africa.
}

Correspondence to Professor C A Wright, Discipline of Anatomical Pathology, PO Box 19063, Tygerberg 7505, South Africa; cawr@sun.ac.za

Published: Archives of Disease in Children, January 2010, 95(1):48-50, doi:10.1136/adc.2009.164038.

\begin{abstract}
Fine needle aspiration biopsy (FNAB) offers a simple outpatient technique for specimen collection in child tuberculosis suspects with peripheral lymphadenopathy. To perform FNAB with mycobacterial culture on an outpatient basis requires use of a sterile transport medium to facilitate bedside inoculation, maintain organism viability and reduce contamination risk en route to the laboratory. The mycobacterial yield and time to positive culture following bedside inoculation into standard mycobacterial growth indicator tubes were compared with initial inoculation into an inexpensive "in-house" liquid growth medium. Of 150 FNAB performed, 57 (38\%) cultured Mycobacterium tuberculosis complex. There was one case each with non-tuberculous mycobacteria and Mycobacterium bovis BCG; the remaining 55 being $M$ tuberculosis. Results were concordant in $142(94.7 \%)$ bedside and laboratory inoculation pairs. There was no significant difference in time to positive culture between bedside and laboratory inoculation (16.2 days (SD 0.87) vs 17.1 days (SD 0.85)). Provision of inexpensive specimen transport bottles and practical tuition in FNAB should improve cost-effective diagnosis of tuberculosis at the primary healthcare level.
\end{abstract}


What this study adds

- A simple and inexpensive means to obtain mycobacterial culture from FNAB specimens was developed using an "in-house" liquid growth medium for transport to the laboratory.

- Transporting FNAB material to the laboratory in the "in-house" medium was similar to a commercial medium with respect to culturing mycobacteria and in time to positive culture.

- Similar results were obtained when the "in-house" transport bottle was kept at room temperature for 7 days before culturing for mycobacteria.

Tuberculosis may be extrapulmonary in 10-30\% children,1 and tuberculous lymphadenitis is the most common extrapulmonary manifestation of tuberculosis in endemic areas;2 $5-10 \%$ of children may have tuberculous adenitis in association with pulmonary involvement. 3 This provides an excellent opportunity to obtain bacteriological confirmation using fine needle aspiration biopsy (FNAB).4 FNAB is a simple and safe outpatient procedure that can be performed by nurses in resource-limited settings 5 and provides material for direct microscopy as well as culture and susceptibility testing. Cultures from FNAB specimens in children give a greater yield in a shorter time, compared with conventional respiratory specimens such as gastric washings. 6

Direct bedside inoculation of FNAB specimens is ideal, and excellent results have been obtained with commercial liquid media systems such as mycobacterial growth indicator tubes (MGIT; Beckton Dickinson, Sparks, Maryland, USA).4 This practice, however, is limited by cost and availability.4 We aimed to develop a simple and cheap mycobacterial transport medium and then to compare the bacteriological yield and time to positive culture achieved with direct bedside inoculation into MGIT versus initial use of the transport medium.

\section{Materials and methods}

All adults and children referred to the FNAB Clinic at Tygerberg Hospital, Cape Town, South Africa (June 2007-May 2008) with clinical mycobacterial lymphadenitis and in whom written informed consent to participate in the study was obtained (following ethics approval) were included.

Tuberculosis transport bottles, containing $1 \mathrm{ml}$ aliquots of Middlebrook $7 \mathrm{H} 9$ broth (with $0.2 \%$ glycerol and $0.05 \%$ Tween 80 added) in $10 \mathrm{ml}$ headspace glass vials, were prepared "in house" (Biomedical TB Research Unit by a study scientist, KGPH) in a laminar flow cabinet, sealed with 20 $\mathrm{mm} \mathrm{TFE} / \mathrm{Sil}$ Septa and $20 \mathrm{~mm}$ aluminium open top seals and autoclaved at $120^{\circ} \mathrm{C} / 20$ minutes (fig 1). Unlike the MGIT tube, the transport bottle does not require removal of the lid, minimising the risk of contamination.

FNAB was carried out following standard protocol.4 Two needle passes were performed: from the first, cytology slides were prepared before the syringe and needle were rinsed by withdrawing an aliquot of liquid growth medium into the syringe and then expelling the contents back into the MGIT tube (bedside inoculation or control). After transport to the laboratory within the same hospital, within 2-24 h, the MGIT tube was incubated in a BACTEC MGIT 960 machine for 42 days. 
From the second FNAB pass, slides were prepared for cytology and the syringe and needle were rinsed into the tuberculosis transport bottle. In the laboratory, $0.5 \mathrm{ml}$ was aspirated from the transport bottle, inoculated into a separate MGIT tube (immediate laboratory inoculation) and incubated as above. In a subset of consecutive samples, the transport bottle was held at room temperature $\left(12-30^{\circ} \mathrm{C}\right)$ for 7 days after which subculture was performed as described above (delayed laboratory inoculation). The delayed inoculation group was designed to mimic the situation of rural clinics where specimens may take some days to reach the laboratory.

PANTA antibiotic mixture (containing polymixin $B$, amphotericin $B$, nalidixic acid, trimethoprim and azlocillin; Becton Dickinson) was added to all MGIT tubes before incubation. All positive MGIT tubes were confirmed to contain acid fast bacilli in the absence of bacterial contamination, followed by accurate PCR speciation.7 The mycobacterial yield and time to positive culture in the respective MGIT tubes were compared using Statistica version 8 software and the $\chi^{2}$ test.

\section{Results}

Over the period of one year 150 FNAB were performed, and $24.5 \%$ of these were in patients less than 16 years, the youngest being 5 months old. Mycobacterium tuberculosis complex was cultured in 57 patients (38\%). There was one case each of non-tuberculous mycobacteria (NTM) and Mycobacterium bovis $B C G$, with the remaining 55 being $M$ tuberculosis, consistent with the profile of disease in our population.3 $48 \mathrm{In}$ the tuberculosis culture-negative patients there were an additional 12 cases (11.4\%) positive on cytology (morphological evidence of mycobacterial infection and identification of the organism). In 17 patients there was a specific diagnosis other than mycobacterial infection, made on cytology, 13 of these were malignant neoplasms.

The results of culture were concordant in 142 (94.7\%) bedside and laboratory inoculation pairs, with eight discordant pairs: three were positive on bedside inoculation only and five were positive on laboratory inoculation only. In one of the discordant pairs, positive on bedside inoculation and negative on laboratory inoculation, there was inadequate material on the cytology slides. There was no significant difference in time to positive culture between the bedside and laboratory inoculation tubes (16.2 days (SD 0.87) vs 17.1 days (SD 0.85)).

There were 31 pairs with matched immediate and delayed laboratory inoculation culture results, of which 29 were concordant (93.6\%). The two discordant pairs showed no growth in the delayed culture, compared with growth in bedside and immediate laboratory cultures; both had required repeated decontamination due to bacterial contamination introduced in the laboratory. There was no significant difference in time to positive culture between the immediate and delayed laboratory inoculation groups (table 1).

\section{Discussion}

Although FNAB has been shown to be an excellent method for obtaining specimens for culture as well as cytology, the method for transporting specimens to the laboratory is rarely specified.9 Mycobacteria need to remain viable during transportation if culture is to be successful and traditional methods for the preservation of specimens, eg, refrigeration10 are not applicable to FNAB specimens in resource-poor settings. In most patients FNAB yields insufficient material to express into a sterile tube, and commercial liquid culture media, eg, BACTEC Myco/F Lytic are recommended only for blood and body fluids.11 Submission of the syringe (with or without the needle attached) directly to the laboratory compromises the quality of the specimen and puts 
healthcare workers at risk. This practice is unacceptable, especially in settings with a high prevalence of multidrug-resistant tuberculosis and/or HIV.

Rinsing the needle and syringe in commercial liquid culture medium, such as MGIT, is very successful in obtaining a positive culture.4 5 However, the widespread distribution of MGIT tubes is limited by issues of cost and storage requirements. These tubes need to be stored in the dark and removal of the cap for inoculation outside the laboratory increases the risk of contamination. This study suggests that subculture from a tuberculosis transport bottle, even after a 7-day delay, gives similar yields in a similar time period, to direct bedside inoculation. The few discrepancies (8/150) noted between the bedside and laboratory cultures may be due to random sampling differences as the tubes were inoculated from separate needle passes.

The transport medium described in this study is inexpensive (MGIT tube US\$4 vs US\$1 tuberculosis transport bottle, 2008 prices in South Africa), easy to prepare "in house", does not require refrigeration as it is stable through a range of temperatures, and does not require removal of the lid for inoculation. Transport of these specimens from rural clinics may follow the same route as other laboratory specimens such as sputum and peripheral blood. The positive culture yield and the time to positive culture from the transport medium, even after 7 days at room temperature, are statistically comparable with direct bedside inoculation into MGIT tubes. The production and distribution of these bottles to clinics and hospitals, combined with practical tuition in the optimal performance of FNAB, will have a positive impact on the cost-effective diagnosis of tuberculosis at the primary healthcare level.

Table

Table 1

The mycobacterial yield and time to positive culture achieved with FNAB and variable timing of MGIT inoculation.

\begin{tabular}{|c|c|c|c|c|c|c|}
\hline \multirow{2}{*}{ Timing of MGIT inoculation } & \multirow{2}{*}{$\begin{array}{l}\text { Total no } \\
\text { specimens }\end{array}$} & \multicolumn{2}{|c|}{$\begin{array}{l}\text { Mycobacterial } \\
\text { culture }\end{array}$} & \multicolumn{3}{|c|}{ Time to positive culture } \\
\hline & & Positive & Negative & $\begin{array}{l}\text { Mean } \\
\text { days }\end{array}$ & SD & $95 \% \mathrm{Cl}$ \\
\hline \multicolumn{7}{|c|}{ Bedside versus immediate laboratory inoculation from transport bottle } \\
\hline Bedside & 150 & $\begin{array}{l}53 \\
(35.3 \%)\end{array}$ & $\begin{array}{l}97 \\
(64.7 \%)\end{array}$ & 16.2 & || 0.87 & $\begin{array}{l}16.8 \text { to } \\
20.3\end{array}$ \\
\hline Immediate laboratory & 150 & $\begin{array}{l}55 \\
(36.7 \%)\end{array}$ & $\begin{array}{l}95 \\
(63.3 \%)\end{array}$ & 17.1 & 0.85 & $\begin{array}{l}15.7 \text { to } \\
19.1 \\
\end{array}$ \\
\hline \multicolumn{7}{|c|}{ Subset-matched pairs: delayed versus immediate laboratory inoculation from transport bottle } \\
\hline Delayed laboratory (day 7) & 31 & $\begin{array}{l}11 \\
(35.5 \%)\end{array}$ & $\begin{array}{l}20 \\
(64.5 \%)\end{array}$ & $\mid 17.1$ & $\mid 1.36$ & $\begin{array}{l}14.1 \text { to } \\
20.1\end{array}$ \\
\hline $\begin{array}{l}\text { Immediate laboratory (day } \\
\text { 0) }\end{array}$ & 31 & $\begin{array}{l}13 \\
(41.9 \%)\end{array}$ & $\mid \begin{array}{l}18 \\
(58.1 \%)\end{array}$ & 16.2 & $\mid 1.31$ & $\begin{array}{l}13.3 \text { to } \\
19.2\end{array}$ \\
\hline
\end{tabular}

- $\mathrm{FNAB}$, fine needle aspiration biopsy; MGIT, mycobacterial growth indicator tube. 


\section{Figure}

Figure 1

Tuberculosis transport bottle.

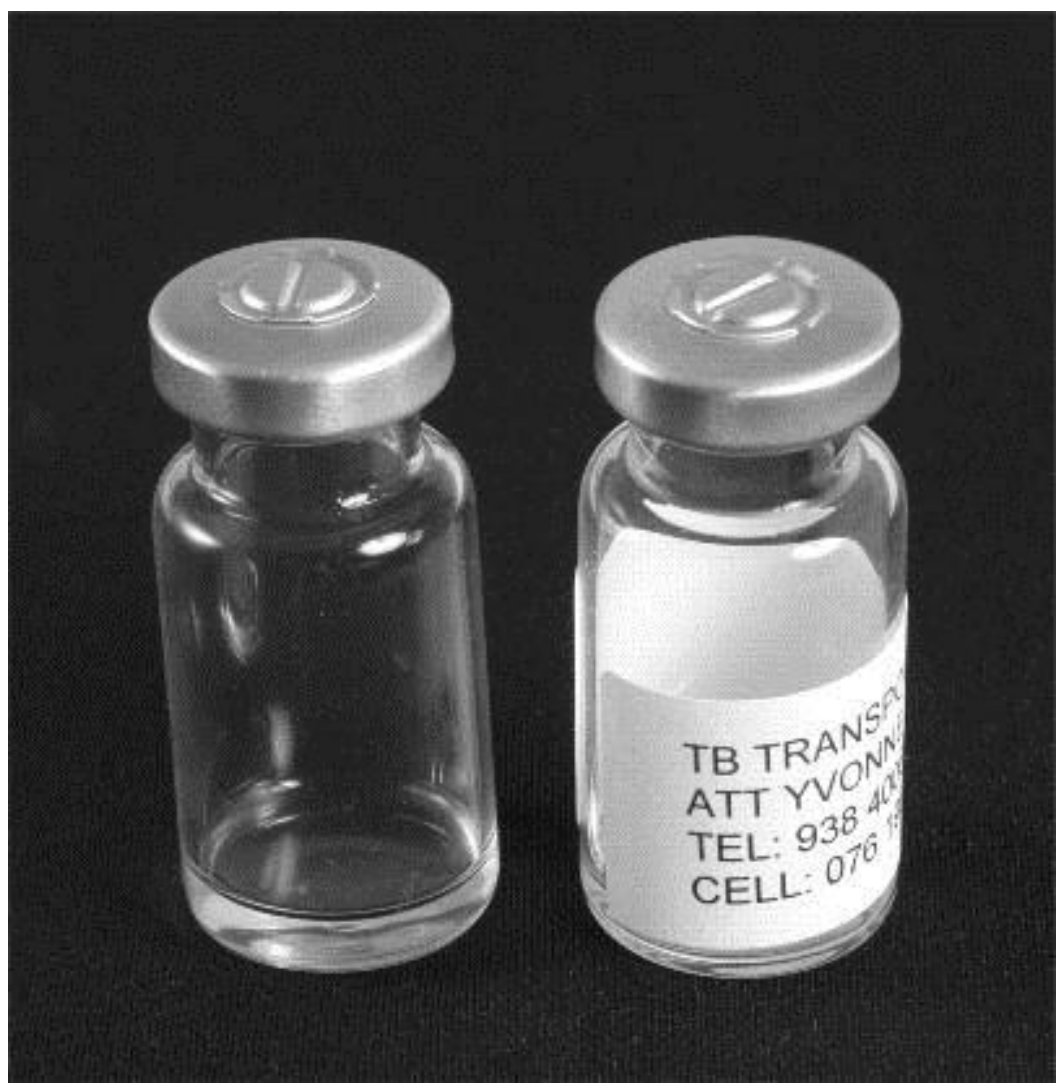

\section{Acknowledgments}

The authors would like to thank Professor Martin Kidd (Stellenbosch University) for statistical analysis. This study is in partial fulfillment of a PhD thesis.

\section{Footnotes}

- Funding: CAW was funded by a grant from the National Health Laboratory Services, South Africa.

- Competing interests: None.

- Provenance and Peer review: Not commissioned; externally peer reviewed.

- Ethics approval: The study was approved by the local ethics committee.

- Patient consent: Obtained.

\section{REFERENCES}

1. Cruz AT, Starke JR . Clinical manifestations of tuberculosis in children. Paediatr Respir Rev 2007;8:107-17.

2. Marais BJ, Gie RP, Schaaf HS, et al. The spectrum of childhood tuberculosis in a highly endemic area. Int J Tuberc Lung Dis 2006;10:732-8. 
3. Marais BJ, Wright CA, Schaaf HS, et al. Tuberculous lymphadenitis as a cause of persistent cervical lymphadenopathy in children from a tuberculosis-endemic area. Pediatr Infect Dis J 2006;25:142-6.

4. Wright CA, van der Burg M, Geiger D, et al. Diagnosing mycobacterial lymphadenitis in children using fine needle aspiration biopsy: cytomorphology, ZN staining and autofluorescence - making more of less. Diagn Cytopathol 2008;36:245-51.

5. Wright CA, Pienaar JP, Marais BJ. Fine needle aspiration biopsy: diagnostic utility in resource-limited settings. Ann Trop Paediatr 2008;28:65-70.

6. Wright CA, Hesseling AC, Bamford C. Fine needle aspiration biopsy-a first line diagnostic procedure in pediatric tuberculosis suspects with peripheral lymphadenopathy? Int J Tuberc Lung Dis 2009; in press.

7. De Wit D, Steyn L, Shoemaker S, et al. Direct detection of Mycobacterium tuberculosis in clinical specimens by DNA amplification. J Clin Microbiol 1990;28:2437-41.

8. Wright CA, van Zyl Y, Burgess SM, et al. Auto fluorescence of mycobacteria on lymph node aspirates a glimmer in the dark? Diagn Cytopathol 2004;30:257-60.

9. Hall GS. Primary processing of specimens and isolation and cultivation of mycobacteria. Clin Lab Med 1996;16:551-66.

10. Kent P, Kubica G. Public Health Mycobacteriology: a guide for the level III laboratory. Atlanta: USDHHS, Centers for Disease Control, 1985.

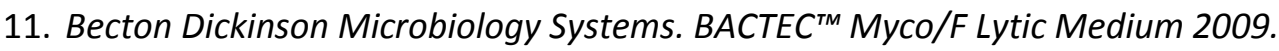
http://www.bd.com/ds/productCenter/442288.asp (accessed 8 Feb 2009). 